\title{
Investigation of Influential Parameters in Deep Oxidative Desulfurization of Dibenzothiophene with Hydrogen Peroxide and Formic Acid
}

\author{
Alireza Haghighat Mamaghani, Shohreh Fatemi, and Mehrdad Asgari \\ School of Chemical Engineering, College of Engineering, University of Tehran, Enghelab Avenue, P.O. Box 11155-4563, \\ Tehran 11155-4563, Iran \\ Correspondence should be addressed to Shohreh Fatemi; shfatemi@ut.ac.ir
}

Received 10 February 2013; Revised 26 April 2013; Accepted 13 May 2013

Academic Editor: Moshe Sheintuch

Copyright (C) 2013 Alireza Haghighat Mamaghani et al. This is an open access article distributed under the Creative Commons Attribution License, which permits unrestricted use, distribution, and reproduction in any medium, provided the original work is properly cited.

\begin{abstract}
An effective oxidative system consisting of hydrogen peroxide, formic acid, and sulfuric acid followed by an extractive stage were implemented to remove dibenzothiophene in the simulated fuel oil. The results revealed such a great performance in the case of $\mathrm{H}_{2} \mathrm{O}_{2}$ in the presence of formic and sulfuric acids that led to the removal of sulfur compounds. Sulfuric acid was employed to increase the acidity of media as well as catalytic activity together with formic acid. The oxidation reaction was followed by a liquid-liquid extraction stage using acetonitrile as a polar solvent to remove produced sulfones from the model fuel. The impact of operating parameters including the molar ratio of formic acid to sulfur $\left(n_{\mathrm{F}} / n_{\mathrm{S}}\right)$, hydrogen peroxide to sulfur $\left(n_{\mathrm{O}} / n_{\mathrm{S}}\right)$, and the time of reaction was investigated using Box-Behnken experimental design for oxidation of the model fuel. A significant quadratic model was introduced for the sulfur removal as a function of effective parameters by the statistic analysis.
\end{abstract}

\section{Introduction}

Deep desulfurization of diesel fuel has become an important issue due to the legislative regulations to reduce sulfur content in most countries (e.g., 15 ppmw since 2006 in the US and $10 \mathrm{wppm}$ since 2008 in the EU) $[1,2]$. Hydrodesulfurization (HDS) is the process most widely used in refineries to remove sulfur compounds from liquid hydrocarbons by transforming them to hydrogen sulfide. This process is catalytically carried out in the presence of hydrogen under severe reaction conditions such as high temperature and high pressure [3].

However, it has been recognized that the achievement of almost zero values of sulfur in fuels has to be done through the combination of HDS process with complementary reactions. Studies reveal that sulfur compounds remaining in diesel fuel after HDS process, at sulfur level lower than $0.1 \%$ wt., are generally thiophene, benzothiophene, dibenzothiophene, and their derivatives. These compounds exhibit low HDS reactivity because of the strict hindrance for the interaction between sulfur and active sites on the catalyst [3].
As a result, to remove refractory sulfur compounds with HDS, more severe operating conditions are needed, including higher temperature and pressure, more active catalysts, and longer residence time, which lead to large hydrogen consumption and reduction of catalyst life. To solve the shortcomings of the HDS process to remove refractory sulfur compounds, several new processes including oxidative desulfurization (ODS), selective adsorption, and biodesulfurization have been developed as alternative or complementary technologies to HDS.

Among these processes, oxidative desulfurization is regarded as one of the most promising methods to face the environmental regulation necessitating ultralow sulfur fuels. The greatest advantage of ODS is mild reaction conditions including ambient pressure and relatively low temperatures. Another crucial feature of ODS is that most refractory sulfurcontaining compounds of HDS process such as dibenzothiophene (DBT) show high reactivity toward ODS process and are easily converted by oxidation. In addition, ODS does not use expensive and corrosive hydrogen. From chemical point 
of view, ODS process consists of two major steps. In the first step, sulfur-containing compounds are oxidized by an oxidizing agent such as hydrogen peroxide to their corresponding sulfones or sulfoxides. At the second stage, these highly polarized products are removed from the fuels by adsorption or liquid-liquid extraction $[4,5]$. Various oxidation systems for ODS process with different oxidants and catalysts have been employed, such as $\mathrm{H}_{2} \mathrm{O}_{2}$ /acetic acid [6-8], $\mathrm{H}_{2} \mathrm{O}_{2}$ /formic acid $[4,9,10], \mathrm{H}_{2} \mathrm{O}_{2}$ /heterogeneous catalysts [11-13], $\mathrm{H}_{2} \mathrm{O}_{2}$ /ionic liquid [14, 15] oxygen/aldehyde [16], ozone/heterogeneous catalysts [17], and tert-butyl hydroperoxide/heterogeneous catalysts [18-20]. Hydrogen peroxide is the most common oxidant for ODS proces, because it is cheap, environmentally friendly, and commercially available. Another advantage of $\mathrm{H}_{2} \mathrm{O}_{2}$ is that it can be joined with various types of catalysts to oxidize sulfur compounds effectively. Zhu et al. [21] performed the oxidation of DBT with hydrogen peroxide, peroxotungsten and peroxomolybdenum complexes as catalyst. In another study, Napanang and Sooknoi [22] examined the oxidative extractive desulfurization of thiophene on titanosilicate-1 with different solvents in the presence of $\mathrm{H}_{2} \mathrm{O}_{2}$. Zhang et al. [23] also examined in situ production of hydrogen peroxide by oxidation of propanol to oxidize thiophene derivative compounds.

Using hydrogen peroxide with an acid has been widely investigated by several researchers. However, to the best knowledge of the authors, there are few studies which are concerned with optimizing the process parameters. The objective of the present work is to develop an efficient system for oxidative desulfurization of model fuel. ODS of dibenzothiophene (DBT) in $n$-octane as simulated fuel was performed in the presence of $\mathrm{H}_{2} \mathrm{O}_{2}$, formic acid, and $\mathrm{H}_{2} \mathrm{SO}_{4}$ as the oxidation system. After the oxidation step, a twostep liquid-liquid extraction with acetonitrile was applied to remove the oxidated sulfur compounds from the model fuel. Influences of operating conditions including $\mathrm{H}_{2} \mathrm{O}_{2} / \mathrm{S}$ molar ratio, acid formic/S molar ratio, oxidation duration time, and medium acidity were examined. The extraction step was carried out at the same conditions in all experiments. Box-Behnken experimental design was implemented as a kind of response surface methodology (RSM) to arrange the experiments and develop a model to explain the relationships of sulfur removal and the studied parameters and finally optimize the operating conditions.

\section{Experimental}

2.1. Materials and Methods. All chemicals used in the present investigation, including $n$-octane (99.9\%), hydrogen peroxide (30\% aqueous solution), dibenzothiophene (DBT), sulfuric acid (95-98\%), formic acid (99\%), and acetonitrile (98\%), were used with no further treatment. Hydrogen peroxide and formic acid were implemented as oxidants, sulfuric acid was used to adjust $\mathrm{pH}$ in the desired range, acetonitrile was exploited as solvent to extract oxidized DBT from the organic phase, $n$-octane was used as a model fuel, and dibenzothiophene served as the model sulfur compound.
An Agilent 6890 plus a gas chromatography system (GC) coupled with a flame ionization detector (GC-FID) using a HP-5 capillary column was applied to detect sulfurcontaining compounds and identify their concentrations in model fuel and product. In this analysis, helium was used as a carrier gas with volumetric flow of $1.1 \mathrm{~mL} / \mathrm{min}$. The sample volume of $2 \mu \mathrm{L}$ was injected into the column without any further solvent dilution. The column temperature was first kept at $100^{\circ} \mathrm{C}$ for 2 minutes and then was heated from 100 to $280^{\circ} \mathrm{C}$ at $10^{\circ} \mathrm{C} / \mathrm{min}$ with a final isothermal period of 10 minutes at $280^{\circ} \mathrm{C}$.

2.2. Procedure. To prepare a solution which could behave as an appropriate sample of real fuel, the model fuel was prepared by dissolving calculated amount of DBT in $n$ octane, in order to obtain a solution with initial sulfur concentration of $250 \mathrm{ppmw}$. A typical set of experiments was designed and carried out as follows.

A $100 \mathrm{~mL}$ three-necked glass was applied as the reactor in the present investigation. This vessel was also equipped with a magnetic stirrer, a thermometer, and a reflux condenser which was placed in a constant-temperature water bath (within $\pm 0.1^{\circ} \mathrm{C}$ ). At first, $50 \mathrm{~mL}$ of the sample fuel was poured into the reactor and heated to the desired temperature. In all experiments, the temperature was set at $65^{\circ} \mathrm{C}$, as the optimal temperature for ODS in the presence of hydrogen peroxide [9]. Then, a proper amount of sulfuric acid (9598\%) was added to the system leisurely, and the mixture was stirred simultaneously. The $\mathrm{pH}$ of the solution was carefully measured by a digital $\mathrm{pH}$ meter and adjusted to the value of 1.5 .

After the solution was stirred vigorously for a few minutes, a suitable amount of hydrogen peroxide (30\% aqueous solution) was added to the reaction mixture immediately. The value of added hydrogen peroxide was chosen in different levels as the experimental design. By addition of hydrogen peroxide, the reaction started. At the same time, an appropriate amount of formic acid (99\%) was introduced to the reactor. Then the mixture was stirred at 700 RPM by a magnetic stirrer during reaction time. After a period of time, the solution was treated by means of liquid-liquid extraction. In each extraction stage, an equal amount of acetonitrile were employed to maintain extraction conditions identical for all cases. The extraction in each step was performed as follows: equal volume of the fuel, after oxidation step, and acetonitrile were decanted into a glass beaker. The beaker was shaken for 5 minutes at room temperature. After separating the aqueous and organic phases and replicating the second stage of extraction at the same condition, the final organic solution was analyzed by GC to detect the amount of DBT in the resulted model fuel. It should be mentioned that because the procedure of solvent extraction was kept identical in all experiments; any difference occurred in DBT detection of the resulted fuel would be related to the oxidation stage in different test runs.

2.3. Parameters. Efficiency of sulfur removal by an oxidative process is affected by various parameters. Some of these parameters were fixed from the previous studies to reduce the 
TABLE 1: Effect of aqueous phase acidity.

\begin{tabular}{lc}
\hline Oxidative system & Sulfur removal (\%) \\
\hline $\mathrm{H}_{2} \mathrm{O}_{2}$ /formic acid & 75 \\
$\mathrm{H}_{2} \mathrm{O}_{2}$ /formic acid/acid sulfuric & 97
\end{tabular}

number of experiments. The amount of sulfuric acid, temperature of reaction, and solvent extraction were fixed according to the prior experiments and derived from the literature. However, the effects of acidity, temperature, and extraction are discussed below before applying for the variables of this work.

2.3.1. Acidity. Sulfuric acid is a very strong acid in aqueous mediums. Using sulfuric acid reduces $\mathrm{pH}$ of the media considerably and enhances DBT conversion significantly. Decomposition rate of hydrogen peroxide to form hydroxyl radicals was reported to be related to the aqueous phase $\mathrm{pH}$ [10]. Due to these results, as aqueous phase $\mathrm{pH}$ value decreases from 5 to 1.5 , DBT conversion enhances about $30 \%$. Improvement in experimental results could be attributed to redox potential. Redox potential of $\mathrm{H}_{2} \mathrm{O}_{2}$ and hydroxyl radicals was influenced by $\mathrm{pH}$ media. At $\mathrm{pH}=0$, redox potential for $\mathrm{H}_{2} \mathrm{O}_{2}$ and $\mathrm{HO}$ was 1.77 and 2.8 , respectively, whereas these values were 0.88 and 2 for $\mathrm{H}_{2} \mathrm{O}_{2}$ and $\mathrm{HO}$ at $\mathrm{pH}=14$, respectively [24]. Therefore, addition of sulfuric acid to the aqueous phase results in $\mathrm{pH}$ reduction and consequently increases redox potential of $\mathrm{H}_{2} \mathrm{O}_{2}$ and hydroxyl radicals. An experiment was designed and performed in order to investigate the effect of aqueous phase acidity on the oxidation of dibenzothiophene with $\mathrm{H}_{2} \mathrm{O}_{2} / \mathrm{HCOOH}$ system. In this experiment, the oxidative desulfurization was done with and without sulfuric acid, while all other parameters were kept identical. Table 1 shows the results of these two experiments. By comparing these results, it is obvious that aqueous phase acidity has a vital effect on sulfur removal. So, due to our preliminary experiments, the $\mathrm{pH}$ of the aqueous phase is maintained in the value of 1.5 in order to obtain better efficiency.

2.3.2. Temperature. Temperature plays an important role in the efficiency of a oxidative system. An increase in the reaction temperature results in a considerable increase in the reaction rate. However, carrying out the oxidation reaction at high temperatures causes thermal decomposition of hydrogen peroxide to undesirable products and reduces the quality of the fuel; therefore, an optimal temperature is required. Temperature and molar ratio of $\mathrm{H}_{2} \mathrm{O}_{2}$ have shown interactive effects $[5,6]$. In this study, the temperature of reaction was fixed at $65^{\circ} \mathrm{C}$ but three levels of $\mathrm{H}_{2} \mathrm{O}_{2}$ molar ratios were examined and analyzed by the experimental design to obtain a better knowledge of the oxidant concentration effect.

2.3.3. Solvent Extraction. During the oxidation reaction, dibenzothiophene (DBT) converts to sulfone and sulfoxide
TABLE 2: The amount and levels of the variables in experimental design.

\begin{tabular}{lcccc}
\hline Variable & Unit & $\begin{array}{c}\text { Low level } \\
(-1)\end{array}$ & $\begin{array}{c}\text { Center point } \\
(0)\end{array}$ & $\begin{array}{c}\text { High level } \\
(+1)\end{array}$ \\
\hline $\mathrm{H}_{2} \mathrm{O}_{2} / \mathrm{S}\left(X_{1}\right)$ & - & 2 & 15 & 28 \\
Time $\left(X_{2}\right)$ & min & 50 & 100 & 150 \\
Facid/S $\left(X_{3}\right)$ & - & 2 & 20 & 38 \\
\hline
\end{tabular}

which are more polar components than DBT due to the presence of sulfur-oxygen bond in their structure. The oxidizedDBT compounds could be removed easily by contacting the treated fuel with a proper polar solvent. This process results in the transfer of oxidized sulfur compounds from model fuel to the more polar solvent which is highly favorable in the thermodynamic point of view. It is because during the transfer of oxidized sulfur compounds, such as DBT sulfones and DBT sulfoxides, from a nonpolar model fuel to a polar solvent, entropy increases sharply. But the important point is to choose a proper solvent in order to optimize the extraction process economically and technically. To choose a suitable solvent, not only the polarity of solvent should be considered, but also the boiling point, density, viscosity, surface tension, toxicity, price, and especially oil-recovery properties should be investigated. Considering these properties, acetonitrile was selected as the appropriate solvent for the extraction of oxidized-DBT compounds. It would be an appropriate choice because of its sulfone removal efficiency, oil recovery, and easy separation [7]. Furthermore, acetonitrile shows a high extraction activity for the sulfone which is a highly polarized and hydrophobic compound $[6,25]$. On the other hand, a set of experiments was designed in order to validate the results of previous works.

In addition, the amount of DBT in model fuel was measured, before and after extraction to find out the effect of extraction step on removal of DBT and sulfones, and it was revealed that negligible amount of DBT could be removed by the lonely process of extraction, whereas the most part of sulfur removal was due to the extraction of polar sulfones and/or sulfoxides by acetonitrile. Therefore, desulfurization by means of oxidative method followed by extraction should be applied as an appropriate method to treat the fuel [9].

\section{Experimental Design}

A kind of response-surface methodology was used to design the experiments for studying the impact of molar ratios of hydrogen peroxide to sulfur, time of reaction, and ratio of acid formic to sulfur on desulfurization efficiency. The variable parameters, denoted by $X_{i}$, were coded as $-1,0$, and 1 , for the low level, center point, and high level, respectively. A table with 15 test runs was made according to Box-Behnken experimental design for the three significant parameters. Those parameters are indicated in Table 2.

The experiments were performed in a random order to disperse the occurred random error in the whole region of the experiments. In addition, the center-point experiment was 
TABLE 3: Coded and actual levels of variables with corresponding responses (initial sulfur concentration $=250 \mathrm{ppmw}$ ).

\begin{tabular}{|c|c|c|c|c|c|c|c|c|}
\hline \multirow{2}{*}{ Experiment no. } & \multicolumn{3}{|c|}{ Coded level of variables } & \multicolumn{3}{|c|}{ Actual level of variables } & \multicolumn{2}{|c|}{ Responses } \\
\hline & $X_{1}$ & $X_{2}$ & $X_{3}$ & $X_{1}$ & $X_{2}$ & $X_{3}$ & S removal (\%) & $\mathrm{S}(\mathrm{ppm})$ \\
\hline 1 & -1 & -1 & 0 & 2 & 50 & 20 & 95 & 12.5 \\
\hline 2 & 1 & -1 & 0 & 28 & 50 & 20 & 100 & 0 \\
\hline 3 & -1 & 1 & 0 & 2 & 150 & 20 & 85 & 37.5 \\
\hline 4 & 1 & 1 & 0 & 28 & 150 & 20 & 100 & 0 \\
\hline 5 & -1 & 0 & -1 & 2 & 100 & 2 & 91 & 22.5 \\
\hline 6 & 1 & 0 & -1 & 28 & 100 & 2 & 100 & 0 \\
\hline 7 & -1 & 0 & 1 & 2 & 100 & 38 & 93 & 17.5 \\
\hline 8 & 1 & 0 & 1 & 28 & 100 & 38 & 100 & 0 \\
\hline 9 & 0 & -1 & -1 & 15 & 50 & 2 & 98 & 5 \\
\hline 10 & 0 & 1 & -1 & 15 & 150 & 2 & 97 & 7.5 \\
\hline 11 & 0 & -1 & 1 & 15 & 50 & 38 & 99 & 2.5 \\
\hline 12 & 0 & 1 & 1 & 15 & 150 & 38 & 100 & 0 \\
\hline 13 & 0 & 0 & 0 & 15 & 100 & 20 & 100 & 0 \\
\hline 14 & 0 & 0 & 0 & 15 & 100 & 20 & 100 & 0 \\
\hline 15 & 0 & 0 & 0 & 15 & 100 & 20 & 100 & 0 \\
\hline
\end{tabular}

TABLE 4: Effects and model coefficients for sulfur removal.

\begin{tabular}{|c|c|c|c|c|c|c|}
\hline \multirow{2}{*}{ Terms } & \multirow{2}{*}{ Mean sum of squares } & \multicolumn{2}{|c|}{ Coefficients } & \multirow{2}{*}{$F$-value } & \multirow{2}{*}{$P$ value } & \multirow{2}{*}{ Significance* } \\
\hline & & Coded value & Actual value & & & \\
\hline Constant & 41.8 & 99.8 & 92.32 & 17.01 & 0.000 & HS \\
\hline$\left(\mathrm{H}_{2} \mathrm{O}_{2} / \mathrm{S}\right) X_{1}$ & 162 & 4.5 & 0.62 & 65.93 & 0.000 & HS \\
\hline (Time) $X_{2}$ & 12.5 & -1.25 & 0.013 & 5.09 & 0.058 & S \\
\hline$($ Facid $/ S) X_{3}$ & 4.5 & 0.75 & 0.042 & 1.83 & 0.218 & PS \\
\hline$X_{1} X_{2}$ & 25 & 2.5 & 0.0038 & 10.17 & 0.015 & $S$ \\
\hline$X_{1}^{2}$ & 45.63 & -3.7 & -0.022 & 18.57 & 0.003 & HS \\
\hline$X_{2}^{2}$ & 4.8 & -1.2 & -0.0048 & 1.95 & 0.02 & S \\
\hline
\end{tabular}

${ }^{*}$ HS: highly significant, S: significant, and PS: possibly significant.

replicated twice to verify the reproducibility of the results. After running the experiments, the left amount of DBT and $\mathrm{DBTO}_{2}$ and the removal efficiency of sulfur was determined and presented in Table 3. Design Expert Software (version 8.0.5) was applied on the results to analyse the variance of the response with respect to the random error (ANOVA) to show the significant parameters and introduce a reliable model.

\section{Results and Discussion}

4.1. Analysis of Variance. The impact of selected three parameters: hydrogen peroxide/S, formic acid/S molar ratio, and time of the reaction was investigated on sulfur removal efficiency, by the response-surface methodology using BoxBehnken statistical design. The weight fraction of sulfur removal was regarded as the response of the experiments. The effect of each variable was analyzed by analysis of variance with $90 \%$ confidence interval. A quadratic model was derived for sulfur conversion related to the variables shown in (1). The calculated Fischer values ( $F$-test) and thereby the probability values $(P$ value) were calculated, and the significance of each parameter was identified for $P$ values of lower than $10 \%$. The parameter X3 with $P$ value of $21.8 \%$ was considered as the possibly significant parameter of the model, as can be seen in Table 4.

ANOVA indicated that there is a linear relationship between the main effects $\left(\mathrm{H}_{2} \mathrm{O}_{2} / \mathrm{S}\right.$, formic acid/S, and time), as well as a quadratic relationship for $\mathrm{H}_{2} \mathrm{O}_{2} / \mathrm{S}$ and existence of binary interaction between time and $\mathrm{H}_{2} \mathrm{O}_{2} / \mathrm{S}$ molar ratio, for sulfur removal in oxidation reaction. The adjusted coefficient of determination was calculated sufficiently high $\left(R_{\text {adj }}^{2}=\right.$ 0.88), which shows a good agreement between the model and experiments. The final quadratic equation in terms of significant effects is presented below:

$$
\begin{aligned}
\operatorname{SR}(\%)= & 92.32+0.62 * X_{1} \\
& +0.013 * X_{2}+0.042 * X_{3} \\
& -0.022 * X_{1}^{2}+0.004 * X_{1} * X_{2}
\end{aligned}
$$

The residual analysis of the model is presented in Figures 1(a), 1(b), and 1(c). Figure 1(a) presents a standardized residual plot of the model response and experiment versus run order. The residuals are dispersed randomly around a line, and there has not been any outlier and leverage in the experiments. Figure 1(b) represents the normal probability plot of 


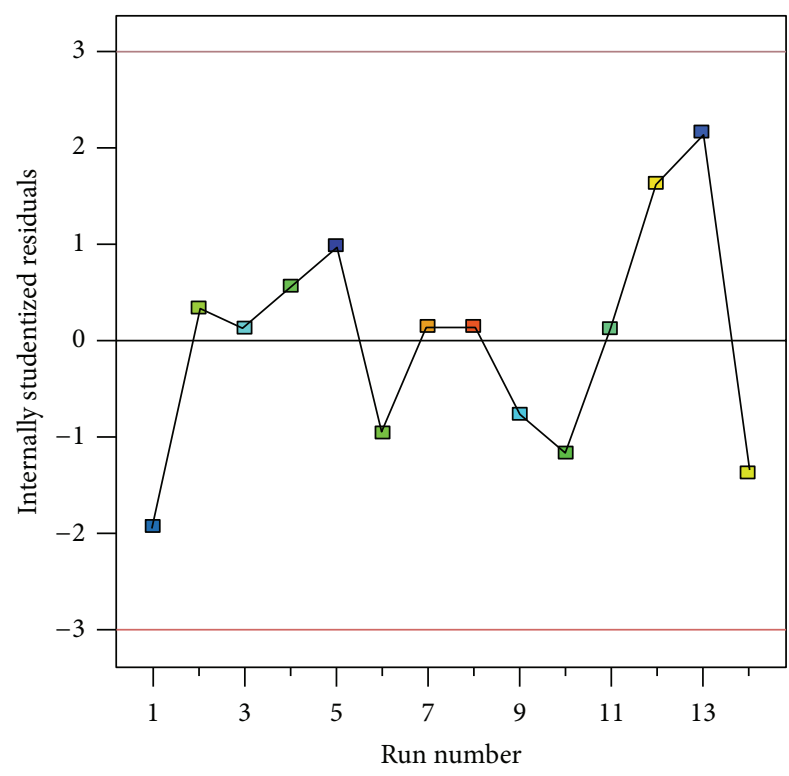

(a)

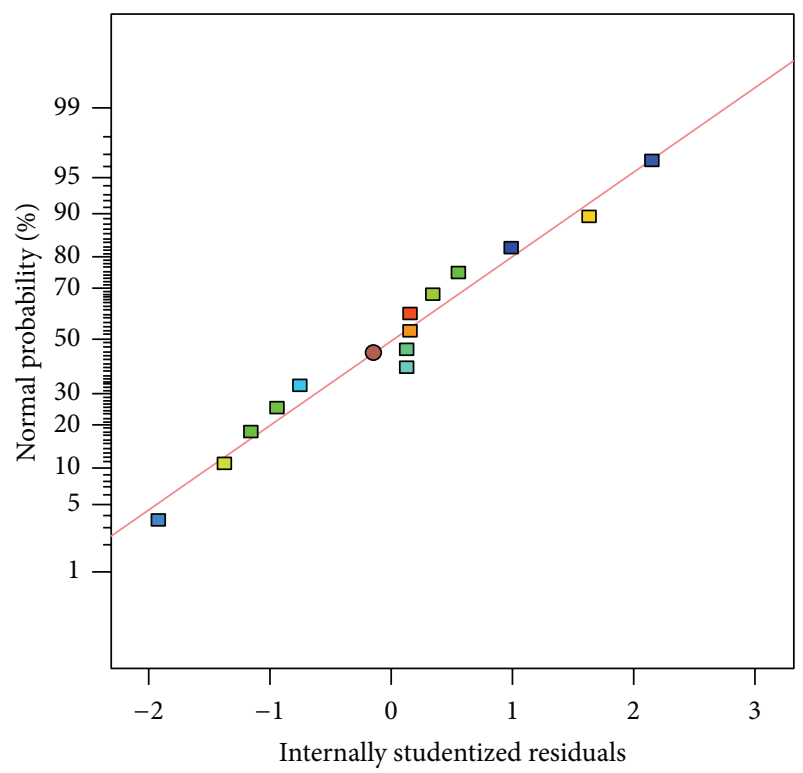

(b)

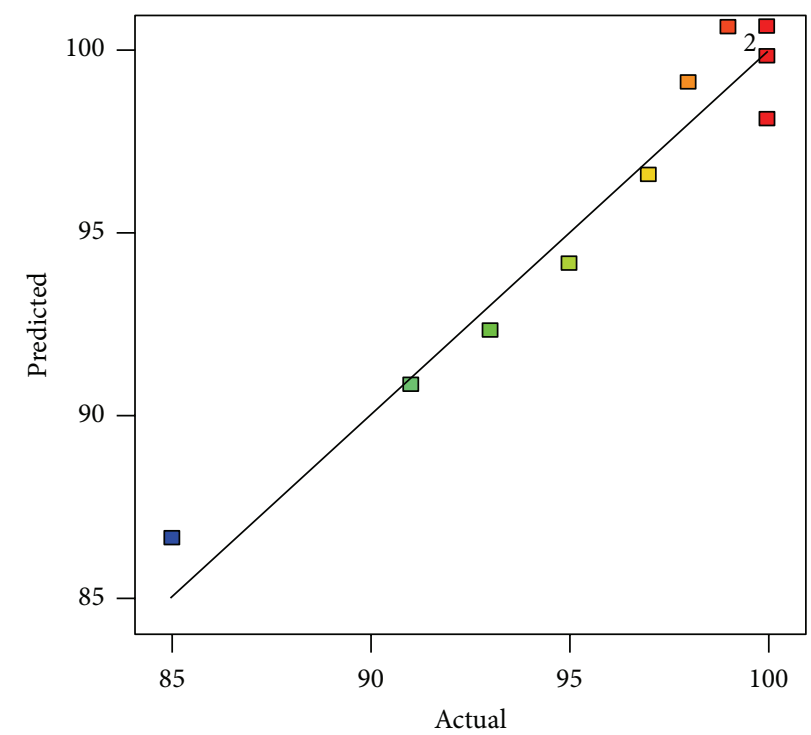

(c)

Figure 1: Residual analysis of the predicted model for sulfur removal. (a) Residual versus the run number, (b) normal probability plot of residuals, and (c) predicted values against the actual values of response.

the residuals, which shows a linear relationship. It proves that the random error is independently and normally distributed. Figure 1(c) presents predicted model versus experimental response that shows a negligible scattering pattern around the bisector [26]. This informative approach implies that constructed model can predict the experiments with fairly well precision.

Operational parameters are also optimized in such a way that oxidizing agent (i.e., $\mathrm{H}_{2} \mathrm{O}_{2}$ ) usage reaches its minimum. This value is selected as an oxidizing agent and is the most valuable and critical operating parameter in the set of experiments.

The optimal operational conditions were determined at $\mathrm{O} / \mathrm{S}=15$, formic acid $/ \mathrm{S}=22$, and $56 \mathrm{~min}$ to achieve
$100 \%$ sulfur removal from the fuel. In order to validate the optimization by statistical methods, an experiment was held in operating conditions which were obtained by statistical methods. Applying optimal conditions resulted in sulfur removal of $100 \%$ which verifies the accuracy of optimization by theoretical methods.

4.2. Influential Parameters. The effect of the studied parameters on sulfur removal is plotted in Figure 2. It is obvious that increasing each parameter can significantly enhance the sulfur removal efficiency. A theoretical discussion will be described in the following sections.

4.2.1. Effect of $\mathrm{O} / \mathrm{S}$ Molar Ratio. The effect of molar ratios of $\mathrm{H}_{2} \mathrm{O}_{2}$ to sulfur is exhibited in Figure 2(a). As indicated 


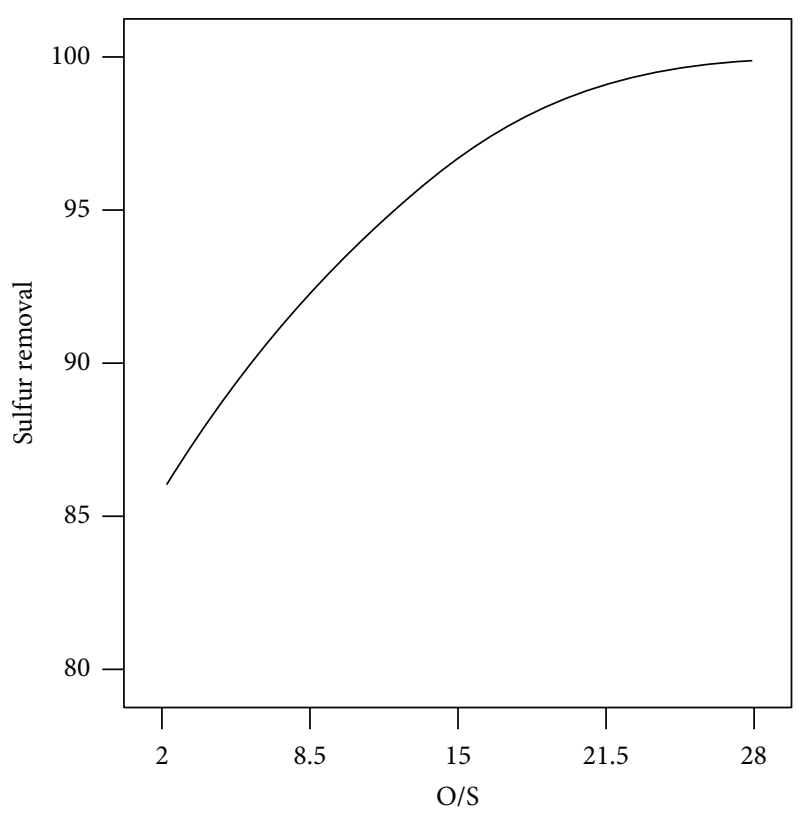

(a)

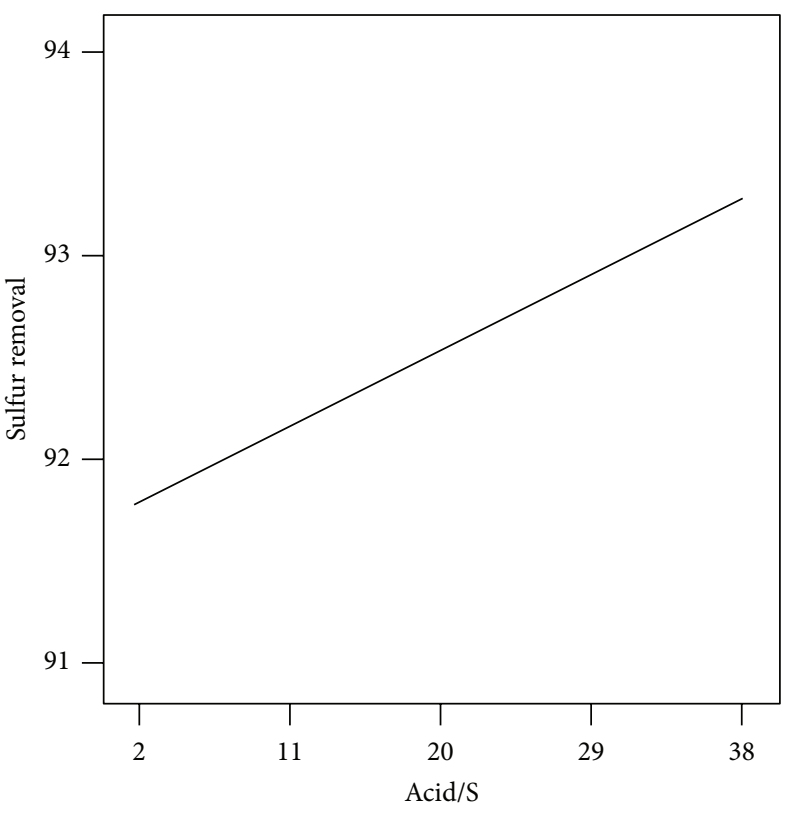

(b)

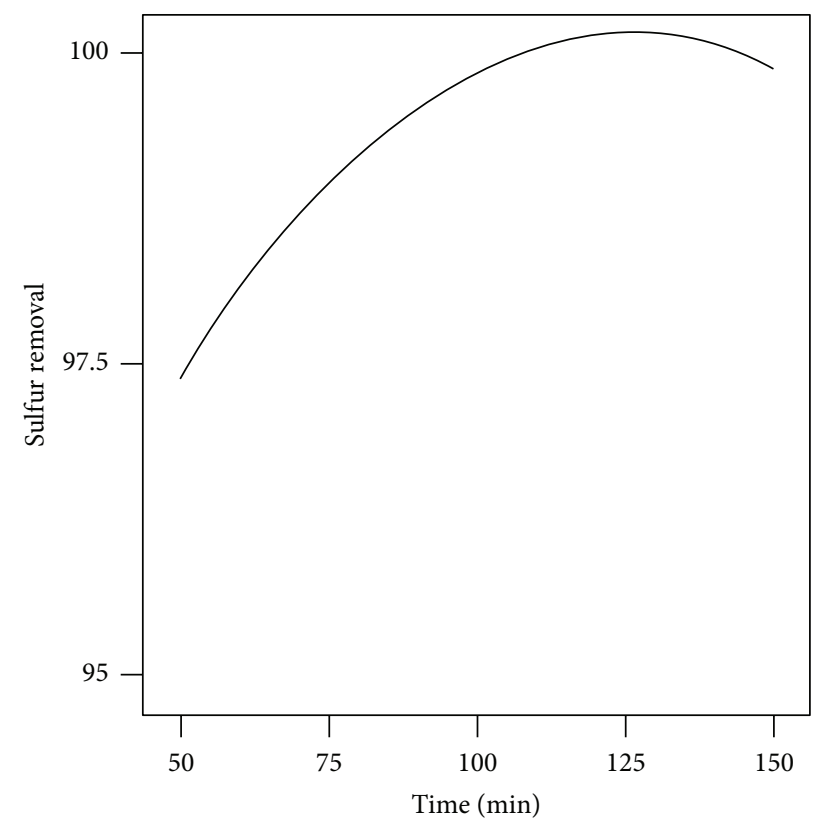

(c)

Figure 2: (a) Sulfur removal as a function of $\mathrm{O} / \mathrm{S}$, acid $/ \mathrm{S}=2$, and time $=150 \mathrm{~min}$. (b) Sulfur removal as a function of acid $/ \mathrm{S}, \mathrm{O} / \mathrm{S}=3$, and time $=100 \mathrm{~min}$. (c) Sulfur removal as a function of time, $\mathrm{O} / \mathrm{S}=28$, and acid $/ \mathrm{S}=2$ (oxidation condition: $T=65^{\circ} \mathrm{C}$; aqueous phase $\mathrm{pH}=1.5$. Extraction condition: $T=22^{\circ} \mathrm{C}$; solvent-to-oil phase volume ratio $=1$; number of extraction stages $=2$ ).

in Figure 2(a), sulfur removal initially increases, as the ratio of the oxidizing agent to sulfur-containing compound rises (i.e., the ratio of hydrogen peroxide to DBT). Then, after a specified level, the amount of oxidizing agent has no considerable effect on the sulfur removal anymore. Best results were obtained at the $n_{\mathrm{O}} / n_{\mathrm{S}}=18$ at $65^{\circ} \mathrm{C}$, and an increase in the $\mathrm{O} / \mathrm{S}$ higher than 18 did not change the sulfur removal significantly. The initial molar $\mathrm{H}_{2} \mathrm{O}_{2} / \mathrm{S}$ concentration determines the initial hydrogen peroxide concentration in the reaction, which has an important impact on the formation of both hydroxyl radicals and performic acid. As mentioned before, according to the literature, these two species are the most effective agents of oxidation. So, it is obvious that by increasing the molar ratio of $\mathrm{H}_{2} \mathrm{O}_{2}$ to sulfur species, DBT conversion increases due to the formation of more hydroxyl radicals and performic acid. On the other hand, results have shown that high molar ratios of oxidizing agent to sulfur compound result in the decomposition of $\mathrm{H}_{2} \mathrm{O}_{2}$ into oxygen molecules, which eventually lead to loss of $\mathrm{H}_{2} \mathrm{O}_{2}$. Definitely this is not a desirable outcome. This means that 


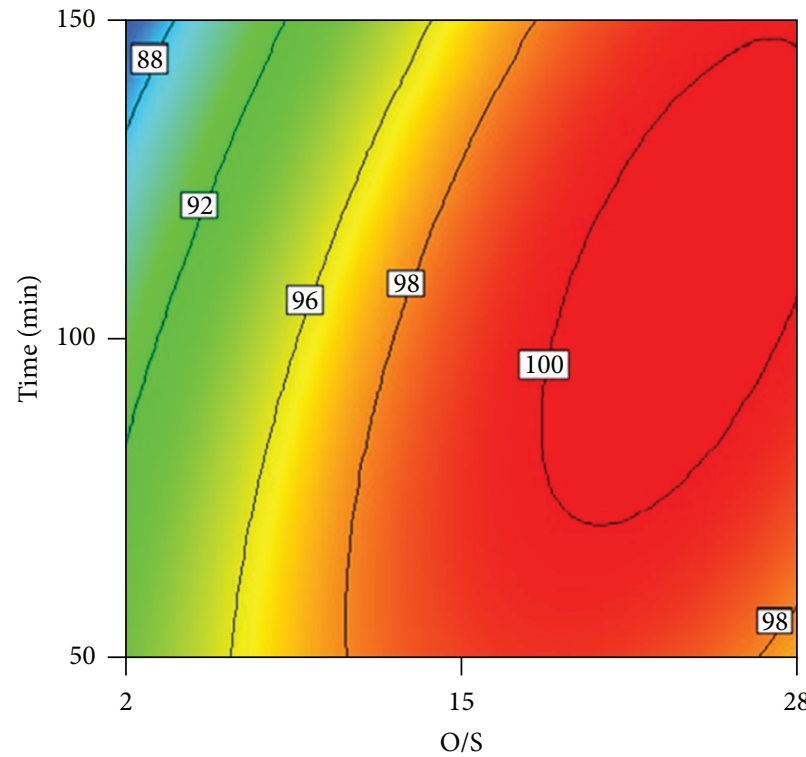

(a)

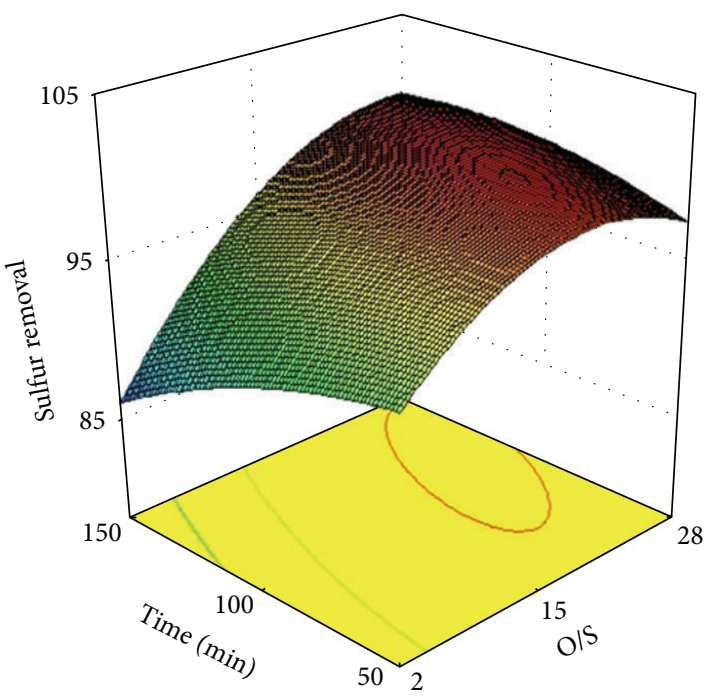

(b)

Figure 3: (a) 2D contour plot and (b) 3D response surface curve of the constructed model displaying sulfur removal at different $\mathrm{O} / \mathrm{S}$ and time. Oxidation condition: $T=65^{\circ} \mathrm{C}$; aqueous phase $\mathrm{pH}=1.5$; acid $/ \mathrm{S}=20$. Extraction condition: $T=22^{\circ} \mathrm{C}$; solvent-to-oil volume ratio $=1$; number of extraction stages $=2$.

greater amount of oxidizing agent beyond the proper level could have no affirmative impact on sulfur removal. The optimum value of $\mathrm{O} / \mathrm{S}$ ratio is strongly dependent upon temperature because temperature can change the amount of decomposition rate significantly. At high temperature (50$\left.70^{\circ} \mathrm{C}\right)$, the $\mathrm{O} / \mathrm{S}$ molar ratio should be great enough $(<10)$ to supply thermal decomposition of $\mathrm{H}_{2} \mathrm{O}_{2}$. However, for a temperature lower than $30-40^{\circ} \mathrm{C}, n_{\mathrm{O}} / n_{\mathrm{S}}$ can be closer to the stoichiometric ratio which is two $[5,6]$.

Therefore, the initial $\mathrm{H}_{2} \mathrm{O}_{2} / \mathrm{S}$ molar ratio should be well controlled. It should be well noted that the stoichiometric ratio of $\mathrm{H}_{2} \mathrm{O}_{2}$ to DBT is two, whereas the excess amount of oxidant is usually required, which is more than the stoichiometric one. Several factors determine the necessity of extra amount of $\mathrm{H}_{2} \mathrm{O}_{2}$, including

(1) mass transfer problems between oil phase and aqueous phase;

(2) thermal decomposition of $\mathrm{H}_{2} \mathrm{O}_{2}$ to water and oxygen;

(3) parallel oxidation reactions of $\mathrm{H}_{2} \mathrm{O}_{2}$ [5].

4.2.2. Formic Acid/S Molar Ratio. Figure 2(b) shows the effect of molar ratios of formic acid to sulfur on the reactions while temperature and $\mathrm{pH}$ values of the solution are maintained at constant values of $65^{\circ} \mathrm{C}$ and 1.5 , respectively. As it was expected, DBT conversion was promoted dramatically by increasing $n_{\mathrm{F} /} n_{\mathrm{S}}$. The results have shown an enhancement in DBT conversion by increasing the ratio of formic acid to sulfur from 2 to 20 , whereas with a further increase in the ratio of formic acid to sulfur, the conversion rate did not increase remarkably. Formic acid could effectively enhance the oxidation because it acts as a cocatalyst in oxidation system and not only decreases the $\mathrm{pH}$ value of the aqueous phase, but also generates performic acid when it interacts with hydrogen peroxide. In addition to the polarization ability of O-O bond, oxidative catalytic feature of formic acid has been reported as an oxidizing agent by giving its oxygen atom to DBT and enhancing its conversion to sulfones and sulfoxides species $[4,5]$. In addition, the small size of the formic acid molecule can facilitate its interaction with sulfur without any steric hindrance of alkyl groups. But like other oxidizing agents, it has an optimum value and increasing its amount to the upper level does not have any effect since its concentration would not act as a controlling parameter in the oxidation system anymore. So beyond an optimum level, increasing formic acid concentration could not enhance the oxidative desulfurization noticeably.

4.2.3. Reaction Time. Figure 2(c) shows sulfur removal as a function of reaction time. As it is indicated in this figure, the results were improved by increasing the time of the reaction initially and then slightly decrease. This could be explained by describing interaction of oxidizing agents as time proceeds. $\mathrm{H}_{2} \mathrm{O}_{2}$ and formic acid can interact with DBT to produce sulfones. This reaction like any other reaction needs enough time to complete and promotes as time goes on. Best results are observed at $125 \mathrm{~min}$. After a particular reaction time, sulfur removal does not enhance by rising reaction time, which means there is an optimum time for oxidation system. Although it can be seen that after the optimum value as time proceeds sulfur removal value decreases, its decline is not sharp at all. This reduction in sulfur removal should be due to loss of hydrogen peroxide and performic acid and their decomposition which accelerates the conversion of oxidized 

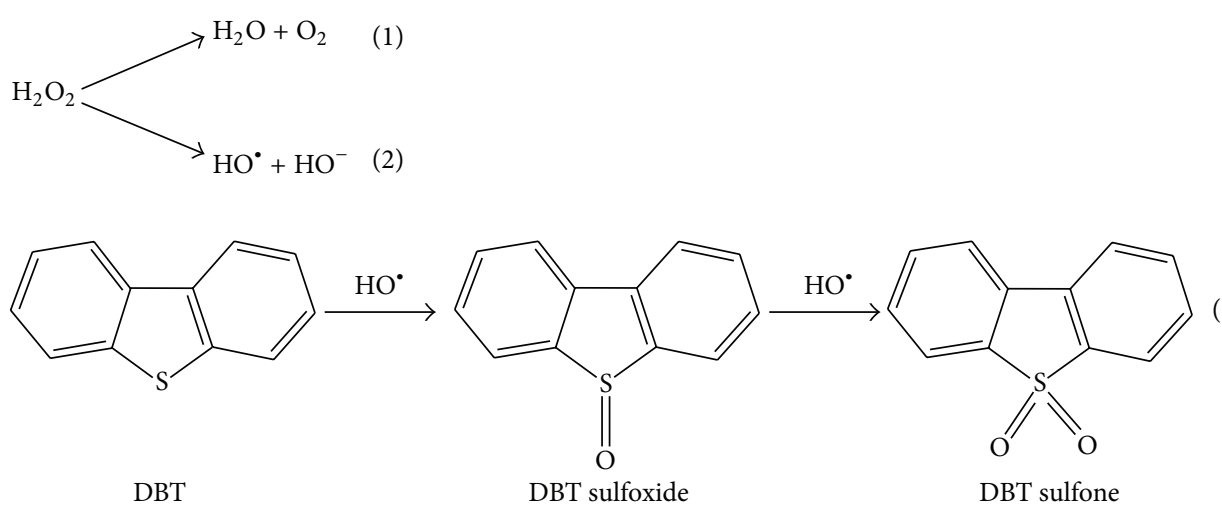

$\mathrm{HCOOH}+\mathrm{H}_{2} \mathrm{O}_{2} \longrightarrow \mathrm{HCO}_{2} \mathrm{OH}+\mathrm{H}_{2} \mathrm{O}$

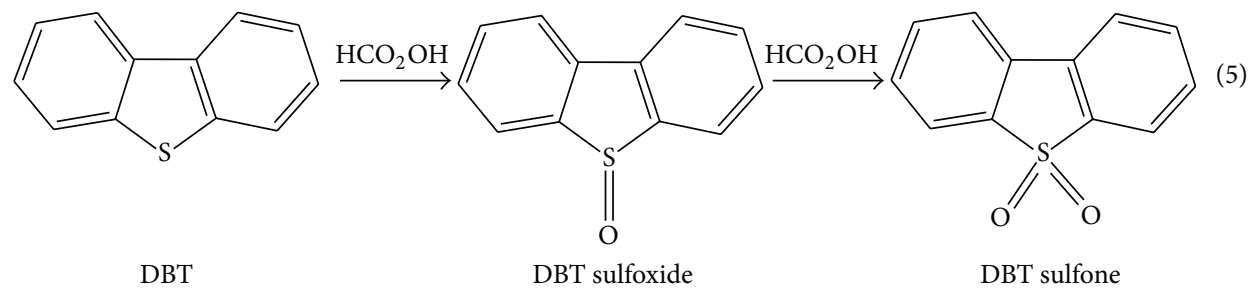

SCHEME 1: ODS reaction of oxidative system and sulfur compound.

sulfur compound to DBT. In other words, both the little amount of oxidizing agents and the long time of reaction result in the loss of all of the oxidizing agents, equilibrium change, and alteration of the reaction media from oxidizing to reducing one. But this trend never could be seen when the main oxidizing agent (i.e., hydrogen peroxide) exists in extra amount in solution. Because in such a condition there are excess amounts of hydroxyl and performic acid radicals over the whole reaction time, and the reaction medium remains as an oxidizing medium. So, carrying out the reaction in a long time especially whenever the oxidizing agent is in a little amount could have no benefit.

4.3. Interaction between $\mathrm{H}_{2} \mathrm{O}_{2} / \mathrm{S}$ Ratio and Time. The contour plot of $\mathrm{H}_{2} \mathrm{O}_{2} / \mathrm{S}$ and time is exhibited in Figure 3(a), which is very helpful in the study of the interactive effects of the factors of the sulfur removal. Figure 3(b) also represents a $3 \mathrm{D}$ plot of sulfur removal as a function of those two parameters. In both figures, the third factor (the ratio of formic acid to sulfur) was kept at a constant level of two. The interaction between time and $\mathrm{O} / \mathrm{S}$ molar ratio arises at different time levels. When a high level of time is applied (150 minutes), sulfur removal increases by increasing $\mathrm{O} / \mathrm{S}$ ratio because $\mathrm{H}_{2} \mathrm{O}_{2}$ and DBT have enough time to react with each other and produce sulfones. On the other hand, when the oxidative system does not have enough time (50 minutes) to interact with sulfur compounds, raising the $\mathrm{O} / \mathrm{S}$ ratio not only causes side reactions involving the thermal decomposition of hydrogen peroxide, but also increases the amount of water in the reaction medium. These results show the balance of two opposing factors (i.e., the excess of oxidant increases the activity, while the water produced from ODS reaction and thermal decomposition hinders the ODS reaction). In addition, it is suggested that in the presence of excess hydrogen peroxide, it acts as a hydroxyl radical scavenger that leads to the generation of less active perhydroxyl radicals [27]. This effect eventually inhibited the oxidation of organic pollutants in the aqueous phase. The best results are found in the region where the ration is limited to $13<\mathrm{O} / \mathrm{S}<24$ at a moderate level of time.

\section{Mechanism of Oxidation}

An appropriate pathway is presented for better understanding the mechanism of oxidative sulfur removal by $\mathrm{H}_{2} \mathrm{O}_{2}$ /acid system. As it is observed in Scheme 1, there are two competitive reactions for $\mathrm{H}_{2} \mathrm{O}_{2}$ that depend on the reaction temperature. The first reaction is the thermal decomposition of $\mathrm{H}_{2} \mathrm{O}_{2}$ that is an undesirable reaction because it increases the amount of water in the system and reduces the concentration of oxidant. Therefore, high temperature is not recommended for this reaction. In Reaction (2), hydroxyl radicals $\left(\mathrm{HO}^{\circ}\right)$ are produced which are strong oxidizing agents. In Reaction (3), DBT(s) are oxidized to their corresponding sulfoxide or sulfones [6].

In presence of acid (e.g., formic acid), $\mathrm{H}_{2} \mathrm{O}_{2}$ and acid interact and produce performic acid $\left(\mathrm{CH}_{2} \mathrm{O}_{3}\right)$ (Reaction (4)). Then, performic acid gives its oxygen to dibenzothiophene and converts DBT into its sulfone (Reaction (5)). 


\section{Conclusion}

This study focused on the oxidative reaction of DBT as a heavy sulfur compound with hydrogen peroxide in the liquid fuel. The model feed containing 250 ppmw sulfur was prepared by dissolving DBT in $n$-octane. A response surface methodology was used to study influential parameters in the removal efficiency of sulfur compounds. Among different types of effective parameters, that is, the fraction of sulfuric acid, temperature, type and fraction of the solvent were kept as sulfuric $/ \mathrm{S}=4$ (mole), $65^{\circ} \mathrm{C}$, volumetric ratio of acetonitrile/oil $=1$, respectively. In addition, the impact of $\mathrm{H}_{2} \mathrm{O}_{2} / \mathrm{S}$, formic acid/S ratio, and time of the reaction were studied by the experimental design to achieve the maximum removal of the sulfur. As a result, a reliable quadratic model was derived to describe the dependency of sulfur removal versus three parameters, quadratic form, and their interactions. Furthermore, the effect of each selected parameter and their interaction were investigated and described comprehensively. Also the operating condition, where best results could be taken, was obtained by statistical analyses (Box-Behnken method). According to the experiments and predicted results, the optimal operating conditions were determined at $\mathrm{O} / \mathrm{S}=$ 15 , formic acid $/ \mathrm{S}=22$, and $56 \mathrm{~min}$ to achieve $100 \%$ sulfur removal from the fuel.

\section{References}

[1] M.-W. Wan and T.-F. Yen, "Enhance efficiency of tetraoctylammonium fluoride applied to ultrasound-assisted oxidative desulfurization (UAOD) process," Applied Catalysis A, vol. 319, pp. 237-245, 2007.

[2] Y. Dai, D. Zhao, and Y. Qi, "Sono-desulfurization oxidation reactivities of FCC diesel fuel in metal ion $/ \mathrm{H}_{2} \mathrm{O}_{2}$ systems," Ultrasonics Sonochemistry, vol. 18, no. 1, pp. 264-268, 2011.

[3] C. Song, "An overview of new approaches to deep desulfurization for ultra-clean gasoline, diesel fuel and jet fuel," Catalysis Today, vol. 86, no. 1-4, pp. 211-263, 2003.

[4] M. F. Ali, A. Al-Malki, and S. Ahmed, "Chemical desulfurization of petroleum fractions for ultra-low sulfur fuels," Fuel Processing Technology, vol. 90, no. 4, pp. 536-544, 2009.

[5] A. M. Dehkordi, M. A. Sobati, and M. A. Nazem, "Oxidative desulfurization of non-hydrotreated kerosene using hydrogen peroxide and acetic acid," Chinese Journal of Chemical Engineering, vol. 17, no. 5, pp. 869-874, 2009.

[6] K.-G. Haw, W. A. W. Abu Bakar, R. Ali, J.-F. Chong, and A. A. Abdul Kadir, "Catalytic oxidative desulfurization of diesel utilizing hydrogen peroxide and functionalized-activated carbon in a biphasic diesel-acetonitrile system," Fuel Processing Technology, vol. 91, no. 9, pp. 1105-1112, 2010.

[7] G. Liu, Y. Cao, R. Jiang, L. Wang, X. Zhang, and Z. Mi, “Oxidative desulfurization of jet fuels and its impact on thermaloxidative stability," Energy and Fuels, vol. 23, no. 12, pp. 59785985, 2009.

[8] W. Liang, S. Zhang, H. Li, and G. Zhang, "Oxidative desulfurization of simulated gasoline catalyzed by acetic acid-based ionic liquids at room temperature," Fuel Processing Technology, vol. 109, pp. 27-31, 2013.

[9] A. M. Dehkordi, Z. Kiaei, and M. A. Sobati, "Oxidative desulfurization of simulated light fuel oil and untreated kerosene," Fuel Processing Technology, vol. 90, no. 3, pp. 435-445, 2009.
[10] G. Yu, S. Lu, H. Chen, and Z. Zhu, "Oxidative desulfurization of diesel fuels with hydrogen peroxide in the presence of activated carbon and formic acid," Energy and Fuels, vol. 19, no. 2, pp. 447$452,2005$.

[11] A. di Giuseppe, M. Crucianelli, F. de Angelis, C. Crestini, and R. Saladino, "Efficient oxidation of thiophene derivatives with homogeneous and heterogeneous $\mathrm{MTO} / \mathrm{H}_{2} \mathrm{O}_{2}$ systems: a novel approach for, oxidative desulfurization (ODS) of diesel fuel," Applied Catalysis B, vol. 89, no. 1-2, pp. 239-245, 2009.

[12] R. Wang, F. Yu, G. Zhang, and H. Zhao, "Performance evaluation of the carbon nanotubes supported $\mathrm{Cs}_{2.5} \mathrm{H}_{0.5} \mathrm{PW}_{12} \mathrm{O}_{40}$ as efficient and recoverable catalyst for the oxidative removal of dibenzothiophene," Catalysis Today, vol. 150, no. 1-2, pp. 37-41, 2010.

[13] J. L. García-Gutiérrez, G. A. Fuentes, M. E. Hernández-Terán et al., "Ultra-deep oxidative desulfurization of diesel fuel bythe $\mathrm{Mo} / \mathrm{Al}_{2} \mathrm{O}_{3}-\mathrm{H}_{2} \mathrm{O}_{2}$ system: the effect of system parameters on catalytic activity," Applied Catalysis A, vol. 334, no. 1-2, pp. 366373, 2008.

[14] W. Zhu, G. Zhu, H. Li et al., "Catalytic kinetics of oxidative desulfurization with surfactant-type polyoxometalate-based ionic liquids," Fuel Processing Technology, vol. 106, no. 1, pp. 7076, 2013.

[15] Y. Nie, Y. Dong, L. Bai, H. Dong, and X. Zhang, "Fast oxidative desulfurization of fuel oil using dialkylpyridinium tetrachloroferrates ionic liquids," Fuel, vol. 103, no. 1, pp. 997-1002, 2013.

[16] S. Murata, K. Murata, K. Kidena, and M. Nomura, "A novel oxidative desulfurization system for diesel fuels with molecular oxygen in the presence of cobalt catalysts and aldehydes," Energy and Fuels, vol. 18, no. 1, pp. 116-121, 2004.

[17] B. Wang, J. Zhu, and H. Ma, "Desulfurization from thiophene by $\mathrm{SO}_{4}^{2-} / \mathrm{ZrO}_{2}$ catalytic oxidation at room temperature and atmospheric pressure," Journal of Hazardous Materials, vol. 164, no. 1, pp. 256-264, 2009.

[18] V. V. D. N. Prasad, K.-E. Jeong, H.-J. Chae, C.-U. Kim, and S.Y. Jeong, "Oxidative desulfurization of 4,6-dimethyl dibenzothiophene and light cycle oil over supported molybdenum oxide catalysts," Catalysis Communications, vol. 9, no. 10, pp. 19661969, 2008.

[19] O. González-García and L. Cedeño-Caero, "V-Mo based catalysts for oxidative desulfurization of diesel fuel," Catalysis Today, vol. 148, no. 1-2, pp. 42-48, 2009.

[20] L. Yang, J. Li, X. Yuan, J. Shen, and Y. Qi, "One step nonhydrodesulfurization of fuel oil: catalyzed oxidation adsorption desulfurization over HPWA-SBA-15," Journal of Molecular Catalysis A, vol. 262, no. 1-2, pp. 114-118, 2007.

[21] W. Zhu, H. Li, X. Jiang, Y. Yan, J. Lu, and J. Xia, "Oxidative desulfurization of fuels catalyzed by peroxotungsten and peroxomolybdenum complexes in ionic liquids," Energy and Fuels, vol. 21, no. 5, pp. 2514-2516, 2007.

[22] T. Napanang and T. Sooknoi, "Oxidative extraction of thiophene from n-dodecane over TS-1 in continuous process: a model for non-severe sulfur removal from liquid fuels," Catalysis Communications, vol. 11, no. 1, pp. 1-6, 2009.

[23] H.-X. Zhang, J.-J. Gao, H. Meng, Y.-Z. Lu, and C.-X. Li, "Catalytic oxidative desulfurization of fuel by $\mathrm{H}_{2} \mathrm{O}_{2}$ in situ produced via oxidation of 2-propanol," Industrial and Engineering Chemistry Research, vol. 51, no. 13, pp. 4868-4874, 2012.

[24] X. L. Zhou, Q. Tan, G. X. Yu, L. F. Chen, J. A. Wang, and O. Novaro, "Removal of dibenzothiophene in diesel oil by oxidation over a promoted activated carbon catalyst," Kinetics and Catalysis, vol. 50, no. 4, pp. 543-549, 2009. 
[25] C. Jin, G. Li, X. Wang, Y. Wang, L. Zhao, and D. Sun, "A titanium containing micro/mesoporous composite and its catalytic performance in oxidative desulfurization," Microporous and Mesoporous Materials, vol. 111, no. 1-3, pp. 236-242, 2008.

[26] Z. Salehi, F. Vahabzadeh, M. Sohrabi, S. Fatemi, and H. T. Znad, "Statistical medium optimization and biodegradative capacity of Ralstonia eutropha toward p-nitrophenol," Biodegradation, vol. 21, no. 4, pp. 645-657, 2010.

[27] Y. Dai, Y. Qi, and D. Zhao, "Effect of various sono-oxidation parameters on the desulfurization of diesel oil," Petroleum Chemistry, vol. 49, no. 5, pp. 436-441, 2009. 

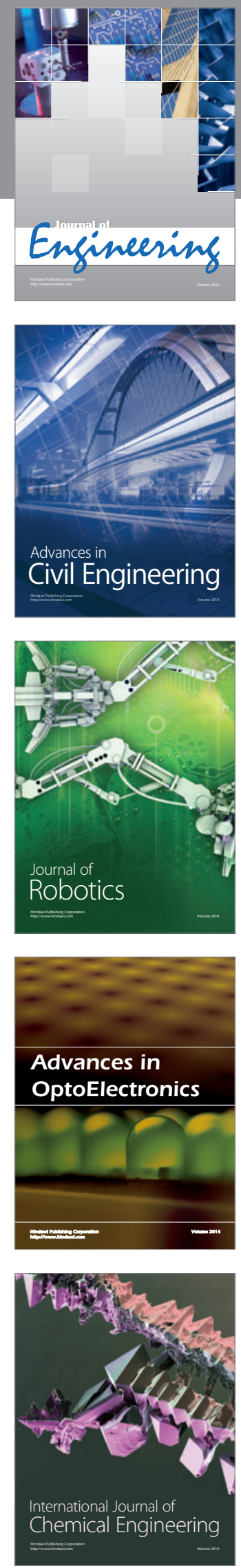

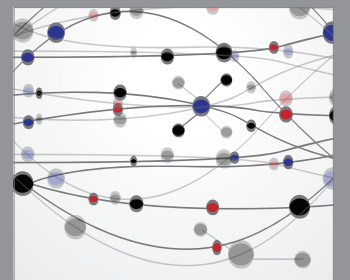

The Scientific World Journal
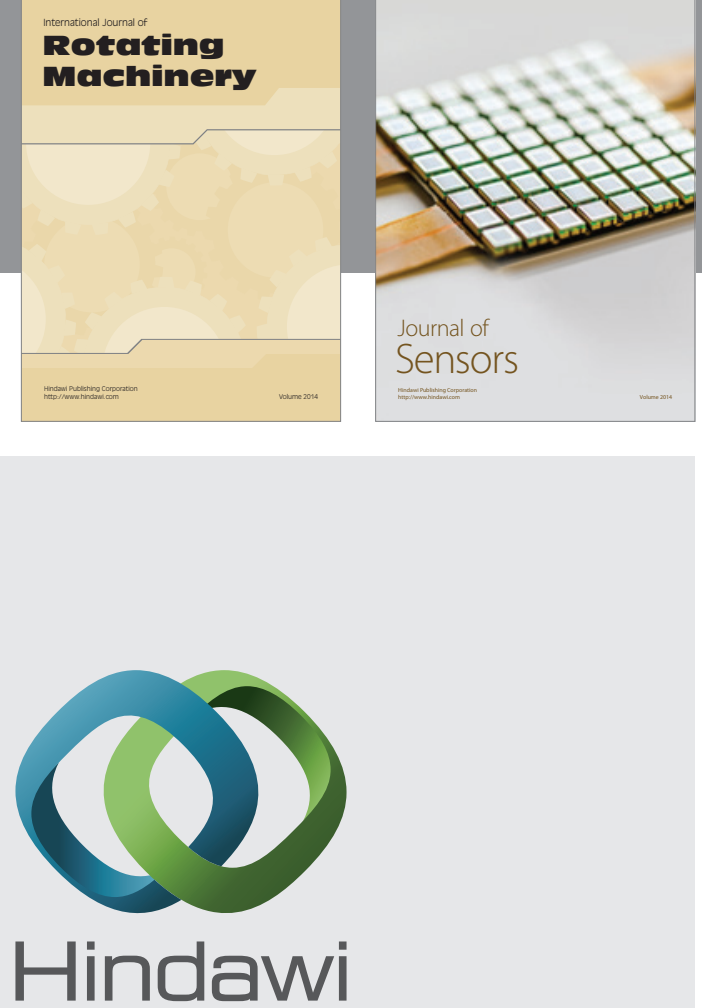

Submit your manuscripts at http://www.hindawi.com
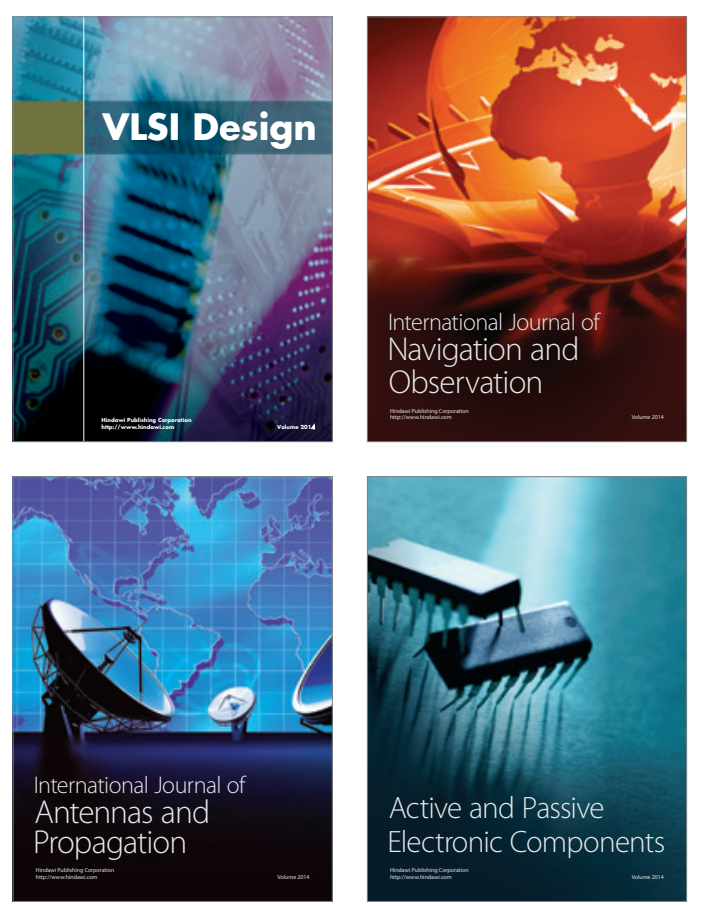
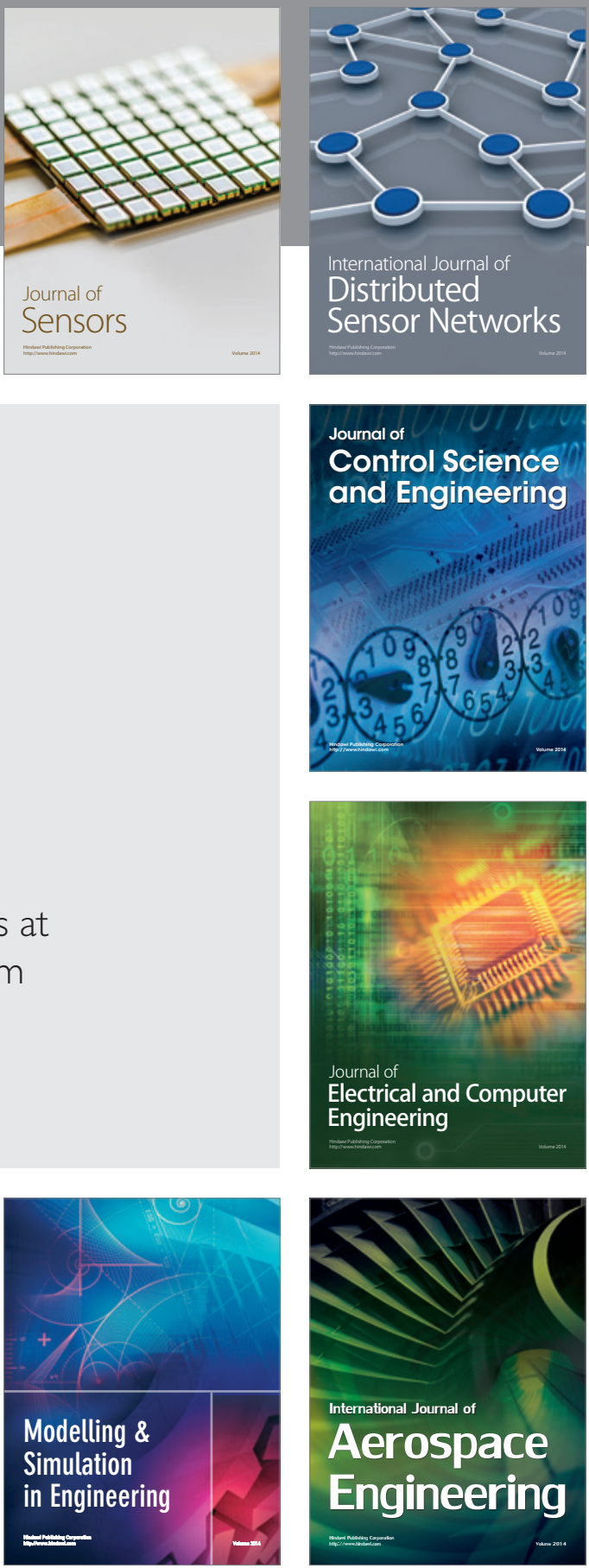

Journal of

Control Science

and Engineering
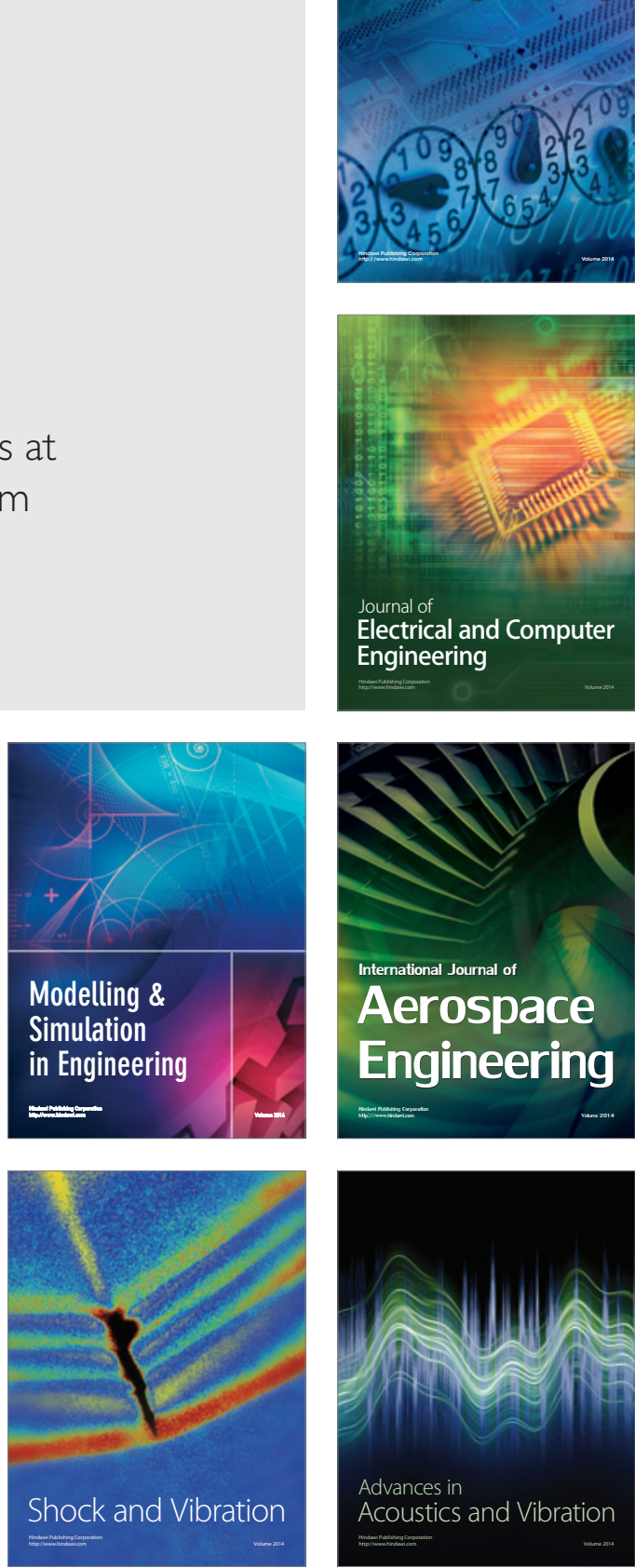